\title{
Glacier de Findelen Avance rapide et construction d'un nouveau captage sous-glaciaire
}

\section{The Findelen glacier}

Rapid advance and construction of a new subglacial water catchment

\section{A. Bezinge}

Ing. SIA - ISF

Chef d'exploitation de Grande Dixence S.A.

Photo A - Glacier de Findelen, vue d'ensemble en été 1982. L'on remarque : les moraines du dernier épisode glaciaire $\sim$ 1820-1840. Le seuil rocheux sur lequel se situe les ouvrages de captage.

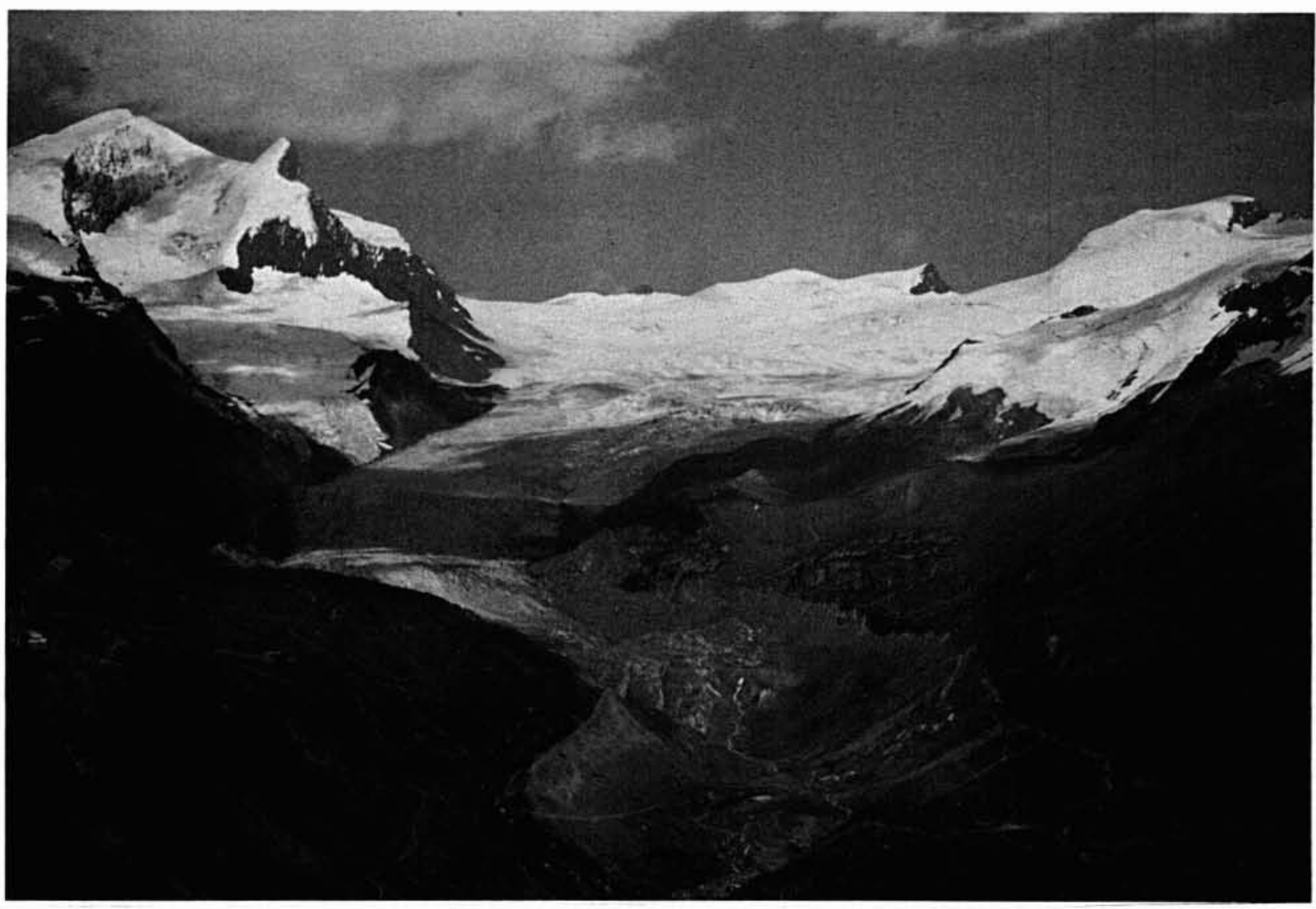

LA HOUILLE BLANCHE/Nº 6/7-1984 
En 1951, lors des projets de captage d'eau pour l'aménagement hydro-électrique de Grande Dixence, on avait prévu de pomper les eaux du glacier de Findelen pour les remonter de la cote 2315 au niveau du collecteur principal, à la cote 2450. Par suite des reculs successifs du glacier, on a pu construire, entre 1962 et 1964 , une prise classique captant l'eau par gravité, à la cote 2475 , tout en dégageant le seuil rocheux de glace morte au bulldozer (Fig. I et photo A).

De 1964 à 1979, le glacier a reculé d'environ $200 \mathrm{~m}$, sans poser de problèmes particuliers d'exploitation de la prise d'eau (Fig. 2).

Dès avril 1980, on s'est aperçu que la rive droite du glacier s'était fissurée selon une ligne de cisaillement longue de $2 \mathrm{~km}$, avec des déplacements différentiels verticaux de plus de $10 \mathrm{~m}$. Devant le front du glacier, on a relevé des plissements du manteau neigeux caractéristiques d'un mouvement rapide (photo $B$ ).

Comme l'équipe de glaciologues du Polytechnicum de Zurich (EPFZ) travaillait sur des forages vers l'altitude 2720 (mesures de pressions hydrostatiques), on a effectué des mesures de déplacements des glaces en altitude et sur le front. Celles-ci ont confirmé une avance rapide de $1 \mathrm{~cm}$ à l'heure au front et $2 \mathrm{~cm}$ vers l'altitude 2720 et ce jusqu'en 1981 (Fig. 3).

La prise construite vers 1962-1964 n'est pas conçue pour résister à une avance glaciaire et risque d'être obstruée par les glaces. Donc, il était urgent pour Grande Dixence d'en construire une nouvelle pouvant fonctionner en sous-glaciaire et ceci avec un maximum de chance de capter les eaux, quelles que soient les épaisseurs du glacier. A titre indicatif, ces eaux turbinées sous une chute de $1890 \mathrm{~m}$ représentent une énergie de plus de $100 \mathrm{mio}$ de kWh.

Cette étude traite des problèmes posés et des solutions trouvées, en liaison avec les ouvrages existants et comprend les différentes observations relevées.

\section{$* *$}

\section{Caractéristiques du bassin versant et du glacier}

Surface totale du bassin

Surface des glaces (1955)

Surface de la zone d'accumulation

Surface de la zone d'ablation

Rapport des surfaces : ablation/accumulation

Limite moyenne du névé à fin septembre $3000-3250 \mathrm{~m}$

Débit de point

Débit max. de captage

Volume estival moyen

Hydrologie spécifique

$24,5 \mathrm{~km}^{2}$
$19,5 \mathrm{~km}^{2}$
$11,5-16,5 \mathrm{~km}^{2}$
$8-3 \mathrm{~km}^{2}$
$0,6-0,2$
$3000-3250 \mathrm{~m}$
$12-13 \mathrm{~m}^{3} / \mathrm{s}$
$11 \mathrm{~m}^{3} / \mathrm{s}$
$24,2 \mathrm{mio} \mathrm{m}$
env. $1 \mathrm{~m} \mathrm{CE}$

Périodes connues de crues du glacier:

— de 1848 à 1865 : forêts renversées à l'altitude 2200

- de 1887 à 1891

- de 1919 à 1920

- recul de 1925 à 1978 env. $1450 \mathrm{~m}$
Avances moyennes des différentes sections du front :

- d'octobre 1978 à octobre 1980

- d'octobre 1980 à octobre 1981

- d'octobre 1981 à octobre 1982

env. $84 \mathrm{~m}$

env. $53 \mathrm{~m}$

env. $48 \mathrm{~m}$

- d'octobre 1982 à octobre 1983

env. $18 \mathrm{~m}$

Analyse des phénomènes hydroglaciaires

\section{Base d'analyse}

Pour trouver les solutions de captage les plus sûres et les plus adéquates, les analyses suivantes sont nécessaires :

\section{Glacier de Findelen :}

- Relever les caractéristiques topographique et morphologique de la zone proglaciaire : lit rocheux ou morainique, croupes rocheuses ou replats, etc.

- Situer les sillons localisés dans le lit rocheux, signes évidents d'écoulements d'eau sous-glaciaires de longue durée.

- Prévoir les zones où les futurs flux glaciaires mettront en compression le glacier avec les effets d'étanchéité bien connus entre glace et lit rocheux ou morainique.

- Refaire l'historique des émissaires observés depuis 50 ans, rive droite et rive gauche (très laborieux).

\section{Glaciers qui présentent des similitudes :}

- Choisir les glaciers qui présentent quelques similitudes morphologiques dans leurs zones frontales : Mt Miné, Bas d'Arolla, Tsidjiore, tous en état de crue; $Z$ 'Mutt en décrue. - Relever les positions des émissaires en fonction de glaciers en crue ou pas.

- Observer les différents phénomènes d'écoulement de l'eau sur des sites sous-glaciaires tels que : galeries de vidange du lac du Gorner, les deux prises du glacier du Weisshorn, prises du glacier du Bondus (Norvège), les nombreux points de contacts du glacier d'Argentière et surtout le captage sous-glaciaire de la Mer de Glace.

Hypothèses sur les écoulements sous-glaciaires et les flux glaciaires (glacier de Findelen)

Lors d'avance du glacier de Findelen, les conditions probables des écoulements, vers l'altitude 2480 , peuvent se définir comme suit :

\section{Zone, rive droite}

Ecoulements sous-glaciaires: en 1963, avec le glacier en décrue, remarqué pour la dernière fois un écoulement sporadique de $0,1-3 \mathrm{~m}^{3} / \mathrm{s}$ entre un flanc rocheux sousjacent et la moraine latérale. C'est pour cette raison qu'un canal avait été construit mais n'a jamais fonctionné par la suite.

Flux glaciaire: lors d'une crue continue du glacier, les glaces se déplacent à l'extérieur d'une courbure générale du glacier de $30^{\circ}$, c'est-à-dire que cette zone de rive droite 


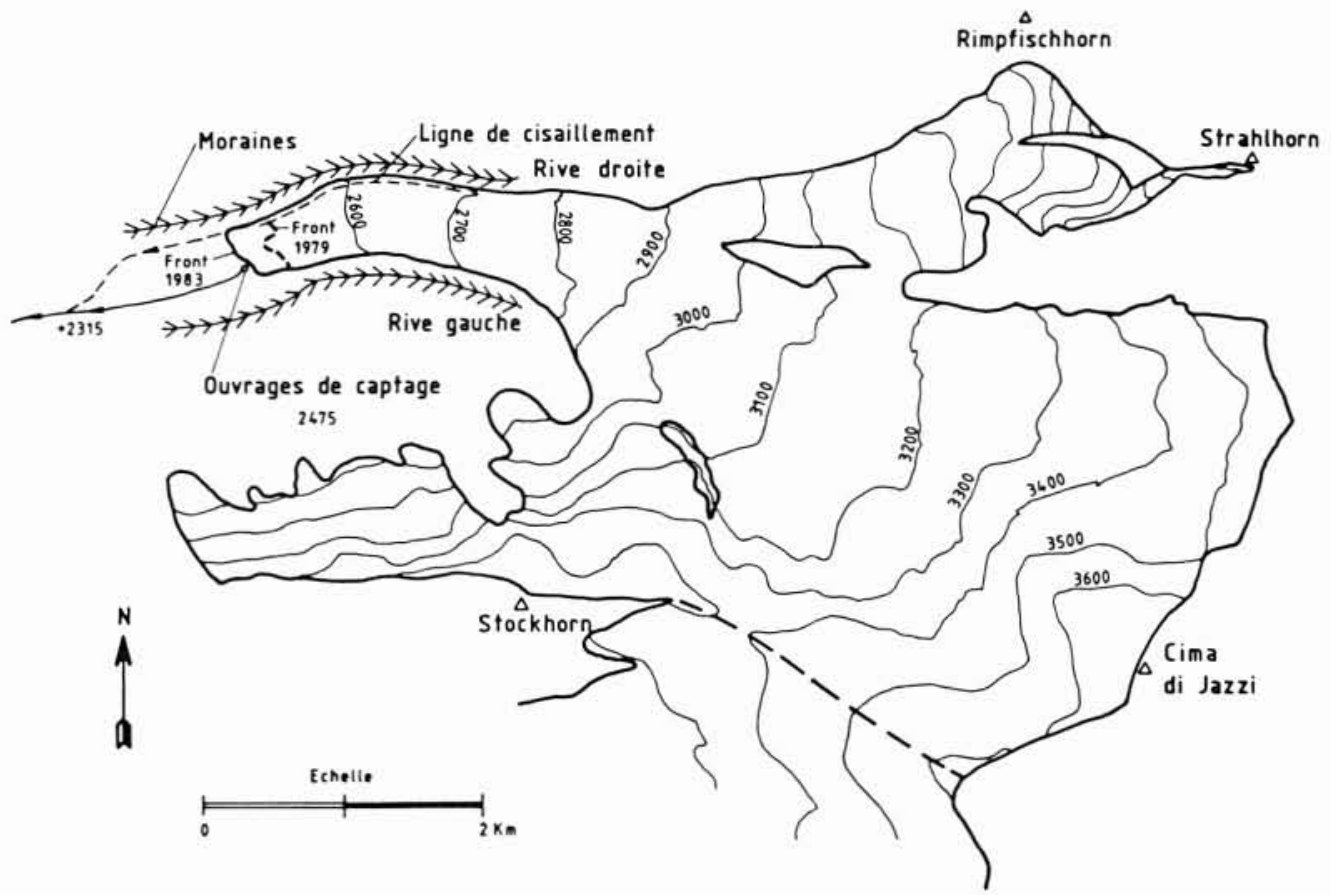

Figure 1 - Glacier de Findelen. Situation et vue en plan.

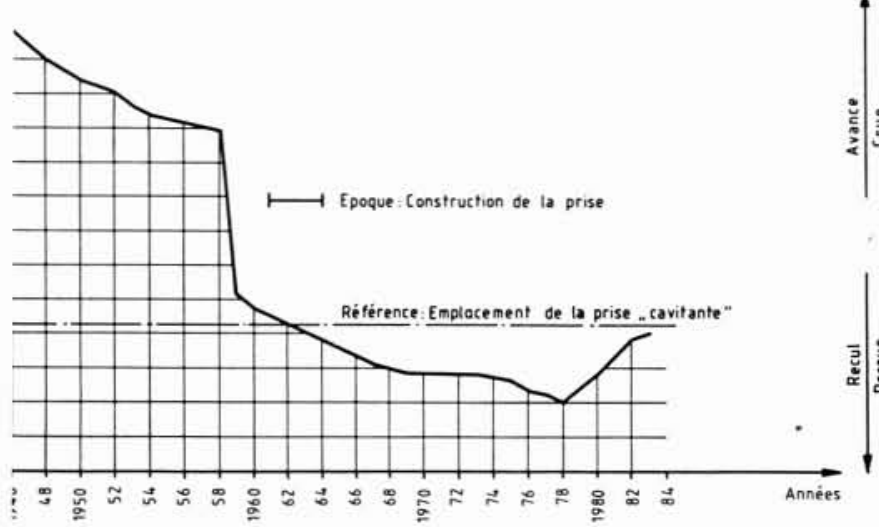

Figure 2 - Glacier de Findelen. Positions du front glaciaire de 1944 à 1983 .

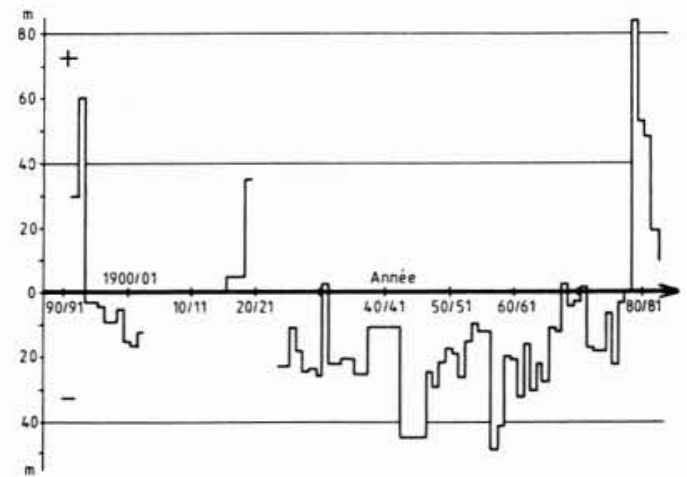

Figure 3 - Glacier de Findelen. Mouvements du front glaciaire de $1892 / 93$ à $1982 / 83$.

Photo B - Rive droite du glacier de Findelen. Ligne de cisaillement en avril 1982.

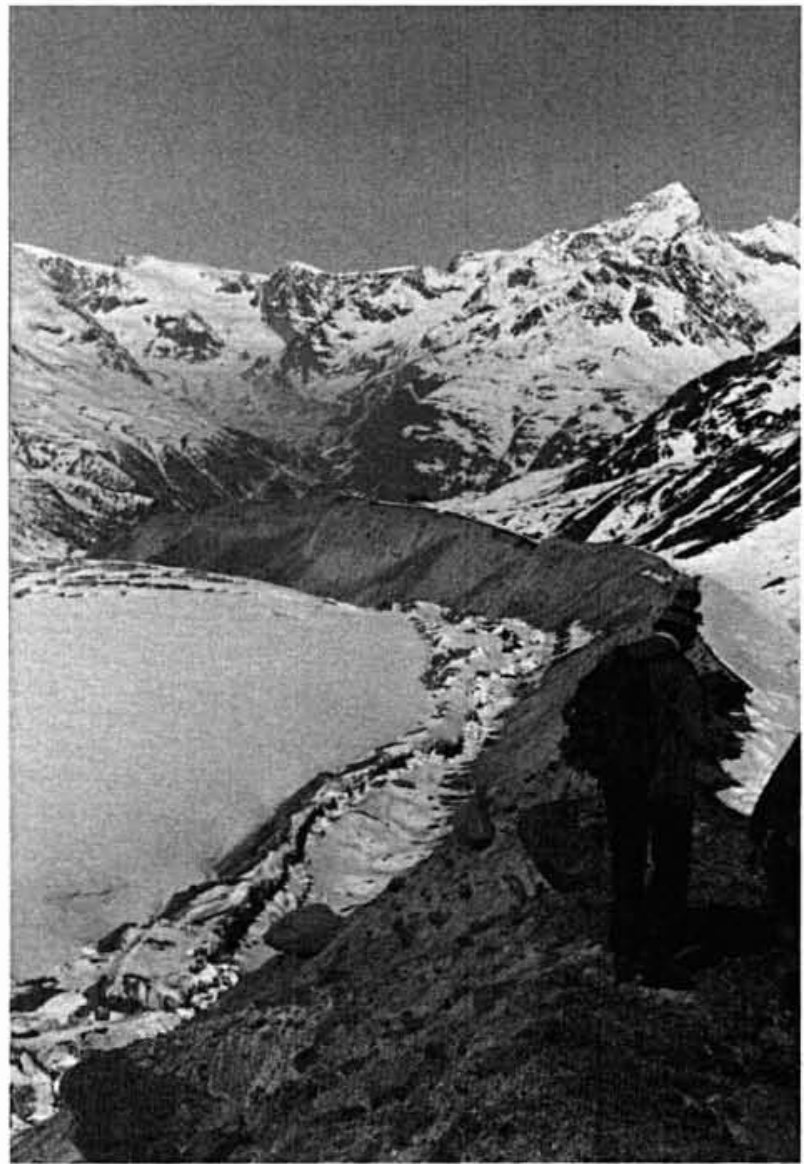




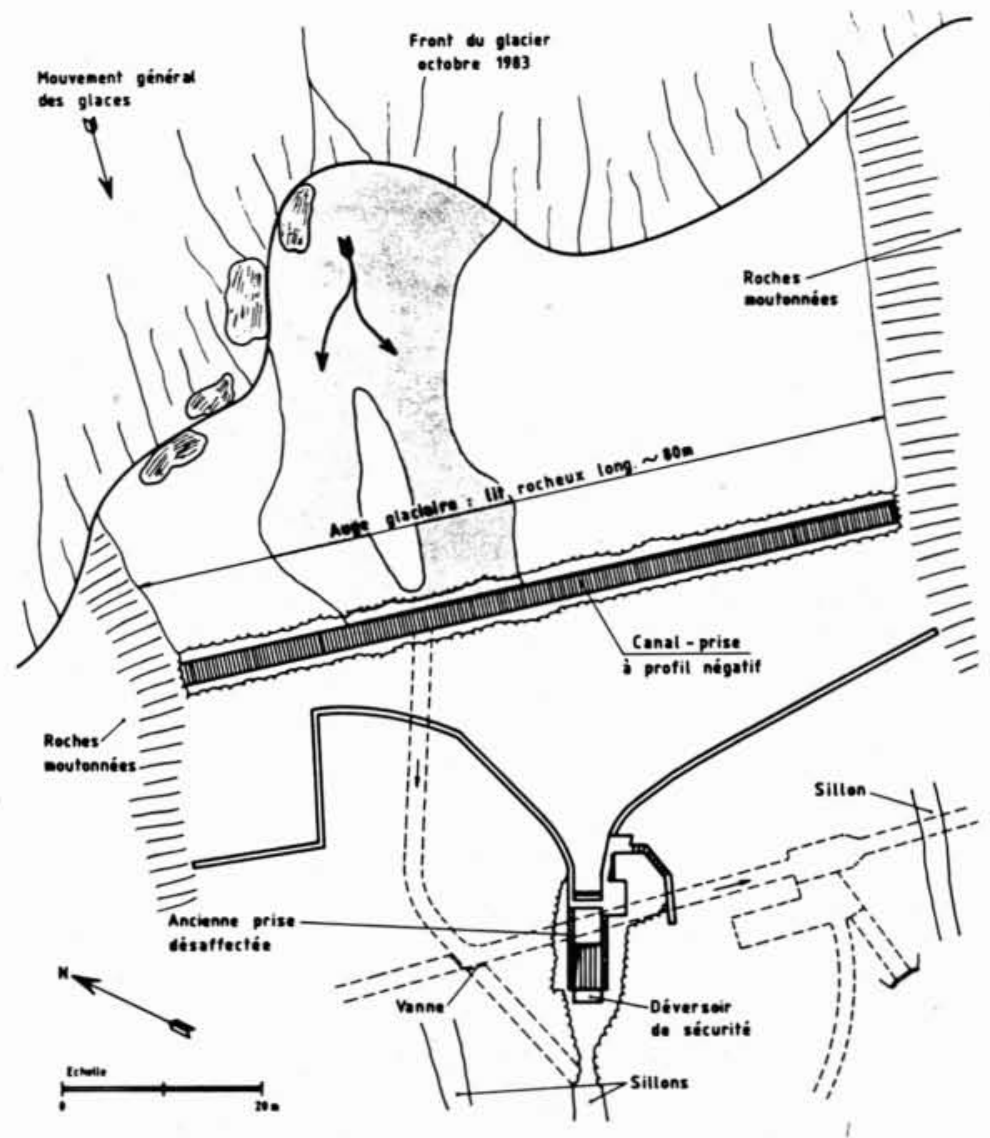

Figure 4 - Glacier de Findelen. Zone rive gauche du front glaciaire, implantation des ouvrages.

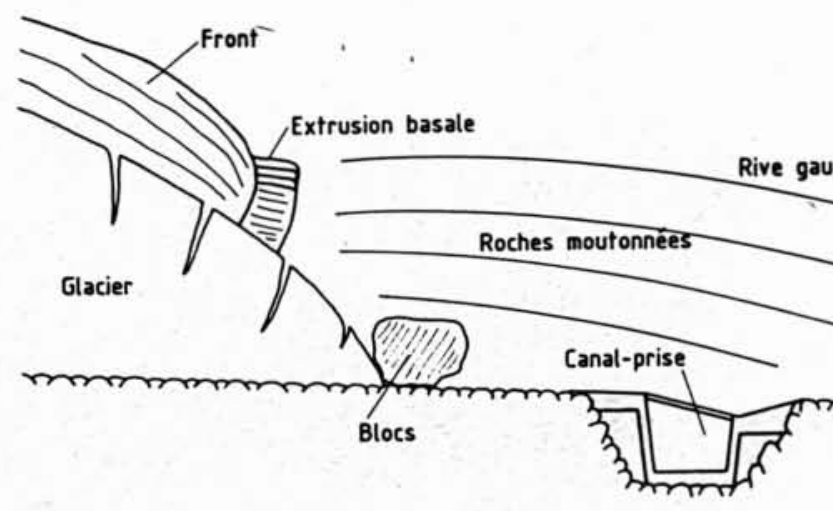

Figure 5-Glacier de Findelen. Profil en long schématique front - ouvrages.

- Ancienne prise désaffectée

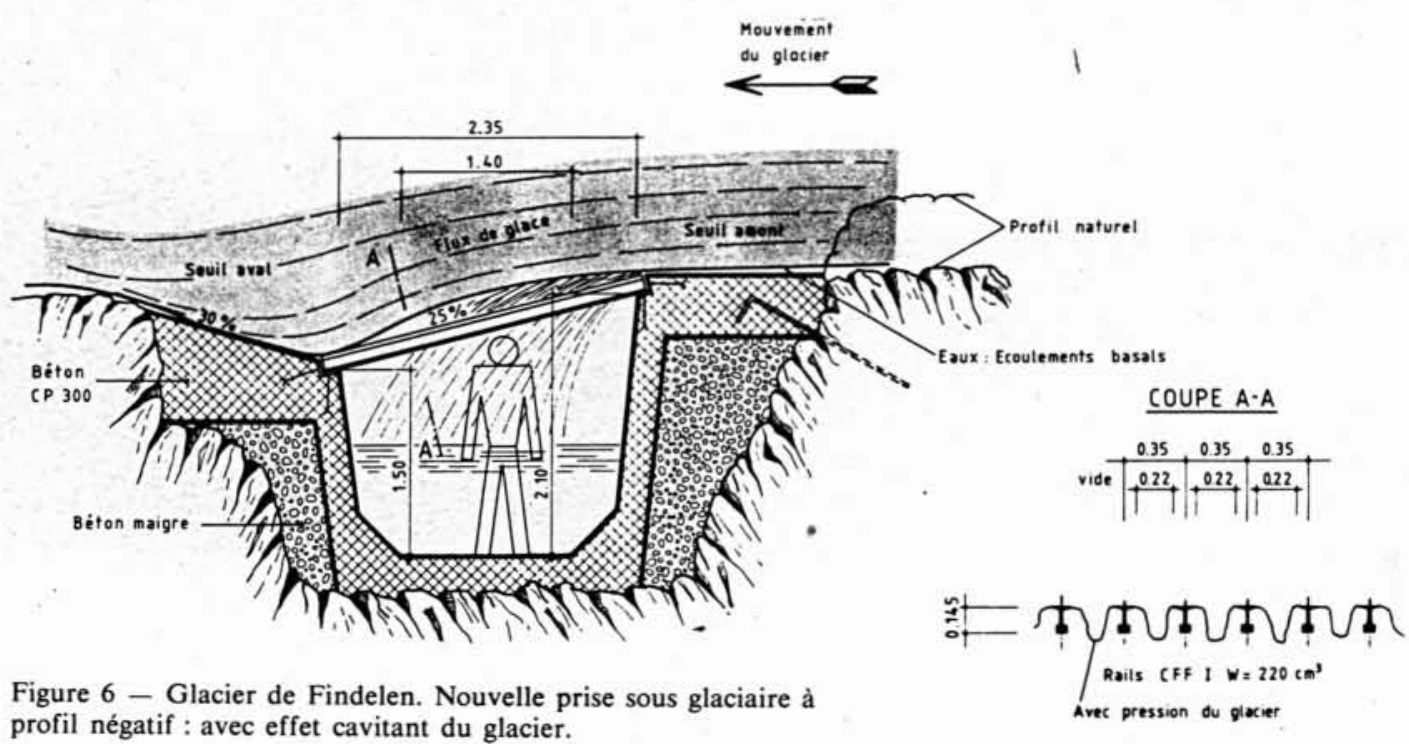
profil négatif : avec effet cavitant du glacier. 
est soumise à une forte compression avec des fluages importants de la glace et des effets d'étanchéités certains, aussi bien intraglaciaires que dans la zone de contacts au rocher et à la moraine. Donc, cette zone n'est pas propice à un captage, d'autant plus que ses écoulements futurs en sont incertains; exemples : Bas d'Arolla, Mont Miné.

En rive droite, la moraine ancienne indique que le glacier de Findelen a atteint une épaisseur maximale de 100-120 m dans cette zone. Avec une épaisseur semblable, le glacier sera à son extension maximale. Il est fort possible que les écoulements préférentiels se produiront au centre du glacier, à l'endroit où les épaisseurs de glaces seront les plus faibles (rognon rocheux, à l'aval duquel on relève d'anciens sillons d'écoulements).

\section{Zone, rive gauche (Fig. I, 4 et 5)}

Ecoulements sous-glaciaires : des écoulements préférentiels et permanents ont créé, en aval d'une large auge rocheuse, des importants sillons, témoins irréfutables de torrents sous-glaciaires. Depuis 1960, lors de l'abandon de cette zone par les glaces, les eaux s'écoulent sous forme de delta large de 10-20 m. En cas de crue du glacier, la topographie locale permet de prévoir que les eaux se fraieront des passages en écoulements diffus ou localisés, entre glaces et roches "moutonnées", limités par l'auge rocheuse; exemple : Bas d'Arolla et Mer de Glace. Sur le sillon principal, la prise ancienne captait les eaux en un point ponctuel, à l'aide de murs bajoyers, et en cas de crue du glacier il était impossible de garantir un captage sûr : destruction des murs, obstruction, écoulements dispersés, etc.

Flux glaciaire : cette zone du glacier est moins soumise aux effets de compression, du fait que l'on se trouve à l'intérieur de la courbure du glacier, c'est-à-dire un peu en dehors de la poussée générale du glacier. Ainsi, cette zone reste soumise au cisaillement et à la flexion et il en résulte une zone crevassée (photographie de 1924). Cette zone présente les chances maximales de capter des eaux, à condition d'adapter un ouvrage aux problèmes sous-glaciaires.

\section{Choix et conception du captage}

D'après les expériences réalisées sur les prises sous-glaciaires de Bis et de Hohberg, à Grande Dixence, et sur celles d'Argentière et de la Mer de Glace, à Electricité de France, nous avons choisi la solution suivante.

\section{Zone, rive droite}

Pas de captage sous-glaciaire pour le moment, au vu des écoulements futurs aléatoires et des difficultés de concevoir avec sûreté une prise dans une zone mixte, lit rocheux avec moraine de fond, et adjacente à une grosse moraine latérale instable comprenant encore de la glace morte.

\section{Zone, rive gauche (Fig. 4, 5 et 6, photos C, D et E)}

D'après les expériences relevées au Gorner et à la Mer de Glace (sous plus de $100 \mathrm{~m}$ de glace), il apparaît que cette zone présente des conditions favorables pour la construction d'un captage à très grande probabilité d'écoulements basal ou sous-glaciaire diffus, variables selon les saisons et les débits. Pour éviter l'obstruction de l'ouvrage projeté et pour capter les eaux avec un maximum de chance, on a conçu et réalisé un canal-prise en rocher, à profil négatif, en utilisant le phénomène de cavitation de la glace en glissement permanent.

L'implantation du canal perpendiculaire au déplacement du glacier se situe dans l'auge rocheuse définie ci-avant. Ce canal transversal dans le lit rocheux est long de $80 \mathrm{~m}$ et situé en amont de l'ancienne prise. Ce canal, à profil trapézoïdal, large de $2,35 \mathrm{~m}$, profond de $1,60-2,20$, présente un seuil aval négatif en béton ou en rocher, avec un plan de reprise du glissement glaciaire, soit une contre-pente de $30 \%$.

Il est protégé par des rails de gros profils montés à l'envers, placés tous les $34 \mathrm{~cm}$, soit un espacement libre de semelle à semelle de $22 \mathrm{~cm}$. La pente de cette grille rudimentaire est de $25 \%$, elle correspond sensiblemęnt à des trajectoires mesurées et relevées sous forme de stries d'érosion sur les rochers moutonnées de la région. Çe phénomène est visible lorsque le glacier quitte un décrochement rocheux : cavitations localisées.

Les rails jouent plusieurs rôles :

- ils empêchent toutes les pénétrations de blocs rocheux ou de glace pouvant obstruer localement la grille. Lors de gros débits, des gros blocs de glace sont arrachés par érosions sous-glaciaires (écoulements turbulents);

- ils créent un frein contre la pénétration des glaces, par création de flux opposés d'écoulements (effet de semelles). Ce phénomène sera particulièrement sensible en hiver, lorsque la résultante des vecteurs de glissement et de fluage a tendance à s'abaisser. Le phénomène a été observé sous la Mer de Glace et à Bis.

La coupe en travers du chenal (fig. 6) montre le profil négatif (seuil aval plus bas que seuil amont) qui crée une cavitation localisée et transversale du glacier et favorise l'intégralité des captages d'eau et la reprise de gros blocs déposés. La contre-pente, en aval, élimine les pertes que l'on pourrait avoir par écoulements parasites sur les rails ou lors d'un écoulement important trop localisé, avec un déversement résiduel au-delà de la prise (effet d'entonnoir). De plus, avec les rails tournés à l'envers, la prise se nettoie facilement des gros blocs de pierre que le glacier peut transporter. Les rails massifs peuvent supporter une poussée accidentelle des glaces.

Les eaux captées par le canal sont reprises par une galerie à radier trapézoïdal et conduites au dégraveleur existant. Une vanne-porte à axe vertical et à commande hydraulique permet, en cas d'incident (obstruction du dégraveleur), d'évacuer les eaux brutes en aval des ouvrages, tout en mettant à sec le dégraveleur. Cette vanne est placée à l'intersection de l'ancienne galerie d'adduction et de la nouvelle. 


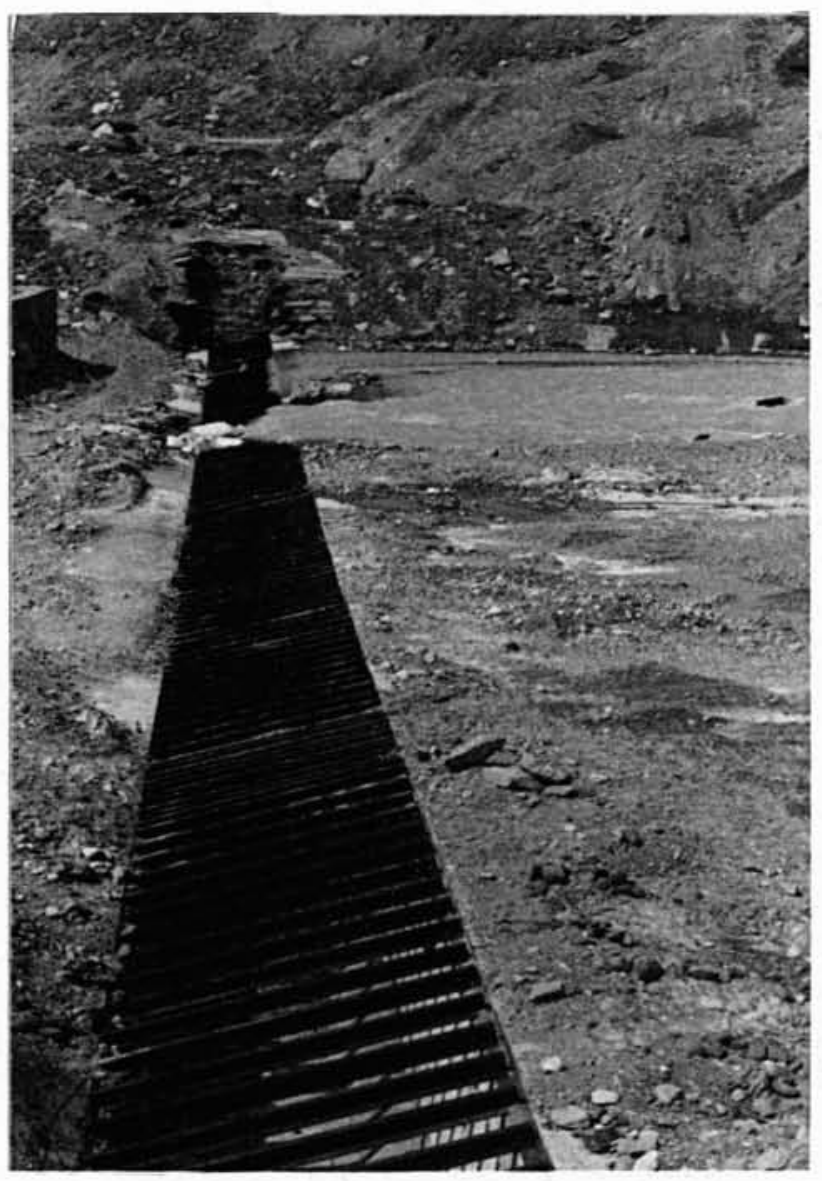

Photo C - Vue générale du nouveau captage avec son canal "cavitant " protégé par des rails de chemins de fers.

Dans le fond, sur le socle rocheux, front du glacier en crue couvert de matériaux morainiques (août 1983).

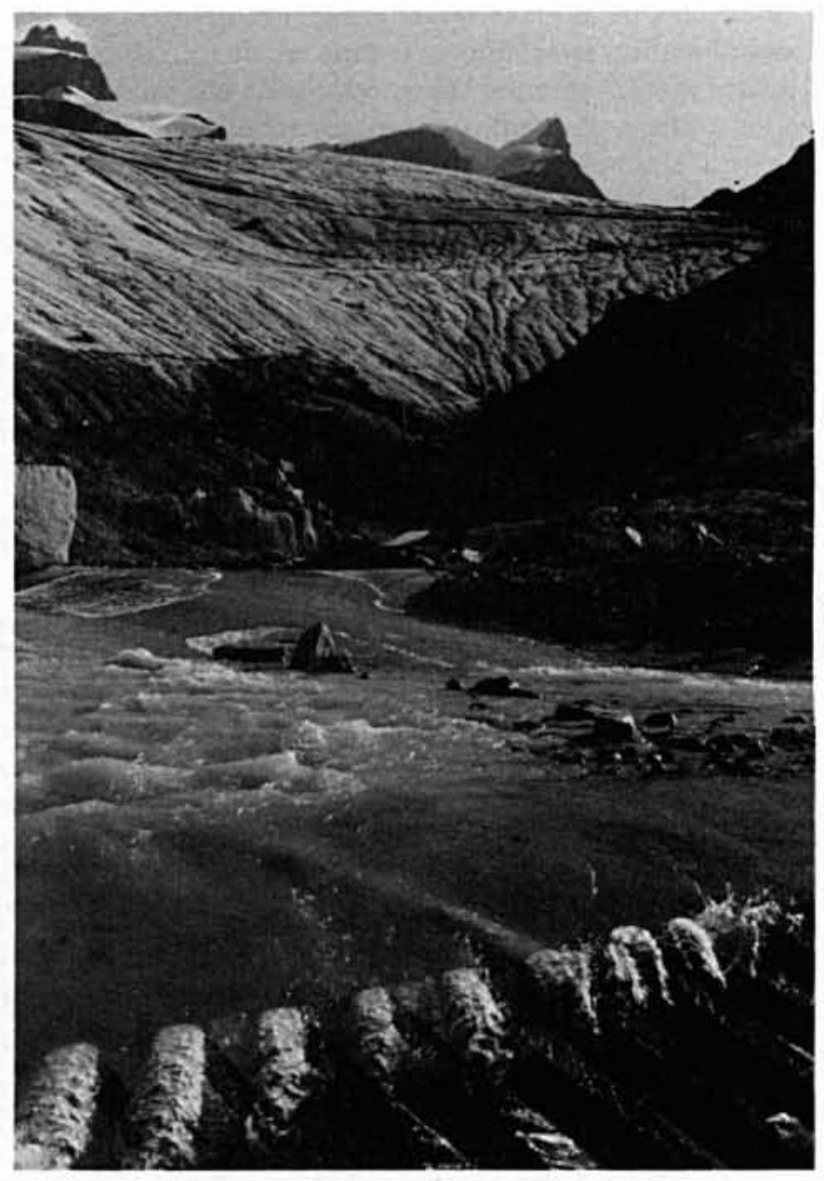

Photo E - Nouveau captage en exploitation et front du glacier, partie centrale (octobre 1983).

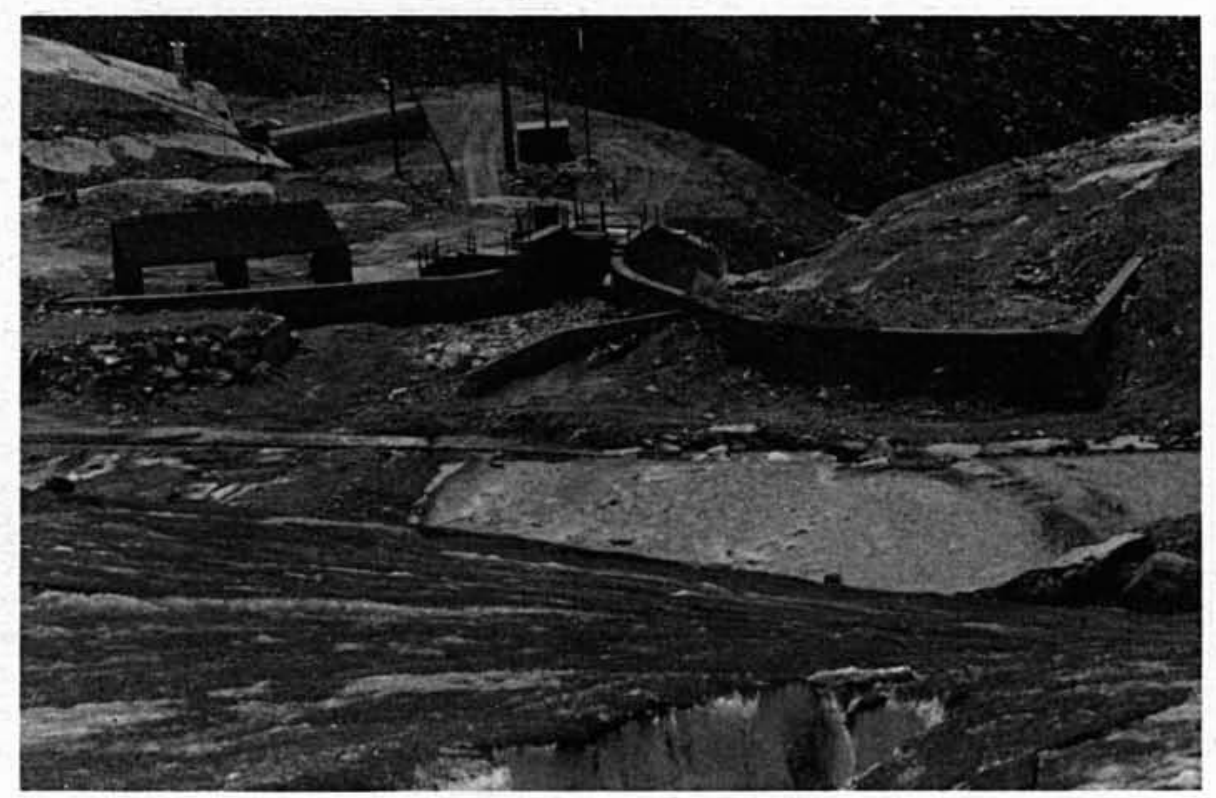

Photo D - Vue générale des ouvrages anciens et nouveaux. A relever l'importance de la masse glaciaire par rapport aux ouvrages. Photographie prise depuis le front glaciaire. 


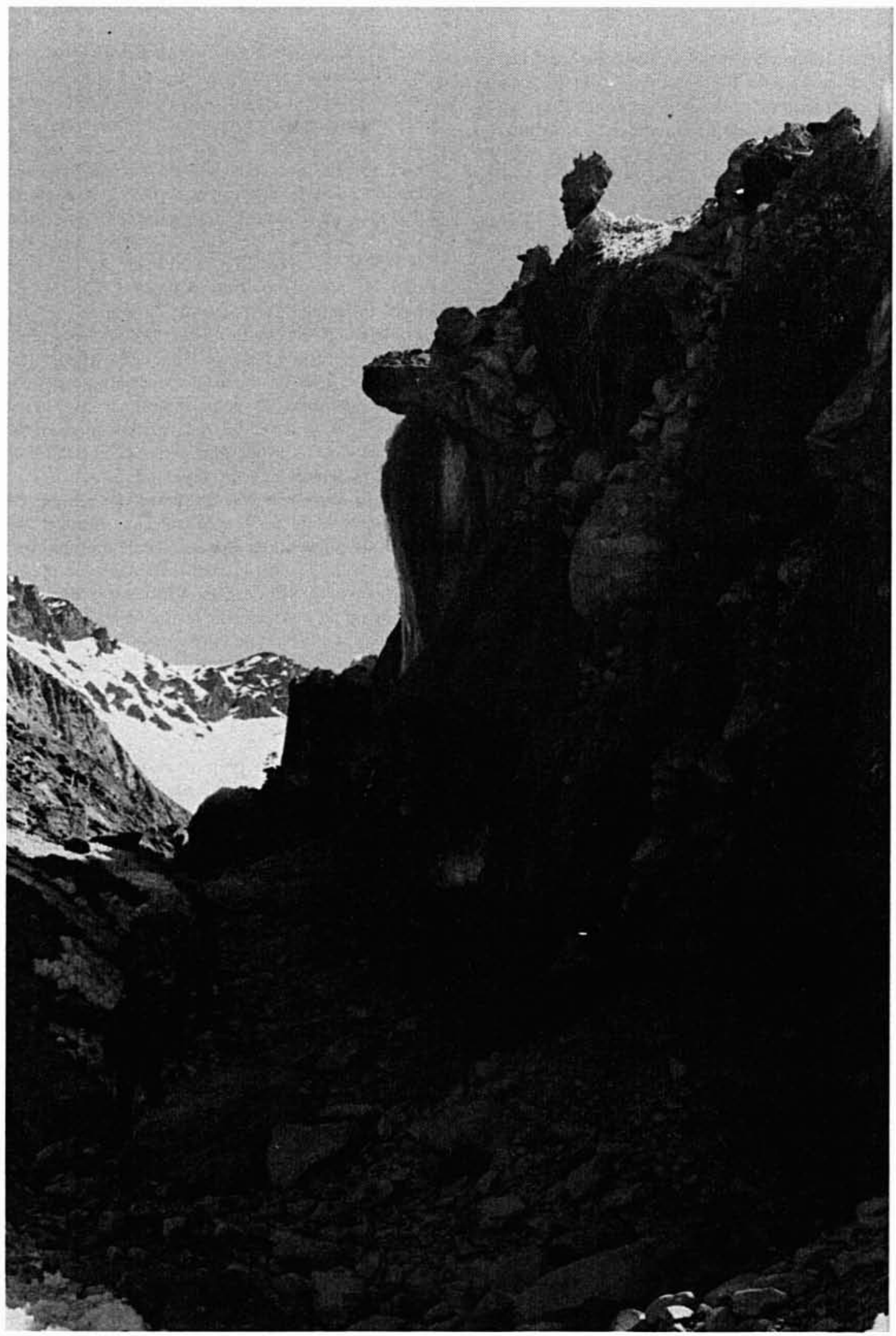

Photo F - Rive droite du glacier : glacier en crue, proche du front.

A l'arrière, rejet latéral de glace (avril 1982) 
Travaux d'exécution

Par suite de gros enneigements, les travaux de construction d'une piste d'accès de $1 \mathrm{~km}$ en moraine n'ont pu débuter que vers le commencement du moịs de juillet 1981. Après le montage des installations de chantiers, les travaux ont démarré et on a pu exécuter, jusqu'à fin octobre 1981, la galerie de dérivation, la tranchée du canal, le bétonnage et la pose des rails sur $45 \mathrm{~m}$ environ.

De grandes difficultés ont été rencontrées lors du minage du chenal qui, par sa géologie et ses plans couches (gneiss Dent Blanche), nous a donné des profils aléatoires qu'il a fallu renforcer par du béton armé avec des remplissages de béton maigre. Les calculs statiques et les ancrages ont été réalisés pour des pressions de glace de l'ordre de $30 \mathrm{~T} / \mathrm{m}^{2}$. Il est certain que les rails fixés sur les fers profilés jouent un rôle d'entretoises qui donnent une rigidité très favorable dans la résistance générale de l'ouvrage.

En été 1982, on a terminé les principaux travaux, non sans mal, du fait des difficultés de déviation des gros débits de fusion, conséquence d'un été très chaud. A la suite de plusieurs mises en charge hydrostatiques du glacier, des blocs de glace de 5 à $6 \mathrm{~m}^{3}$ ont été débités et transités sur l'ouvrage en contruction.

En 1983, la nouvelle prise à ciel ouvert a été utilisée sans difficultés, avec de gros débits. Impressionnés par le front " raide " du glacier et par sa hauteur $50-60 \mathrm{~m}$, on a décidé, à titre de sécurité, de rapprocher les rails et de les tourner à l'envers : semelle en haut. L'été très chaud de 1983 a fourni des débits extrêmes de fusion, avec beaucoup de blocs de glace. Pour limiter les débits captés, on a créé un déversoir temporaire sur le pied aval de l'ancienne prise.

Actuellement, les distances entre le front du glacier et le nouveau canal varient entre 10 et $30 \mathrm{~m}$. Avec les vitesses de glissement de 1983, les temps avant recouvrement varieront de 6 à 24 mois. A relever qu'au centre du glacier, sur la droite du nouveau canal, le front glaciaire est au-delà de l'axe de la nouvelle prise.

\section{Observations particulières (photo B)}

Les phénomènes glaciaires importants observés sont :

- Un plan vertical de cisaillement sur rive droite, long de $2 \mathrm{~km}$, qui se crée en début d'hiver avec des formes maximales en avril-mai, pour s'estomper en été. Ce phénomène s'est produit avec plus ou moins d'intensité de 1980 à 1983. Il a aussi été relevé sur le glacier de Tsidjiore-Nouve et sur l'extérieur de la courbure du glacier.

- Les profils en travers des glaces sur ce plan vertical de cisaillement montrent des remontées de glace de 10 à $20 \mathrm{~m}$, avec un cisaillement vertical, côté moraine, et une pente inclinée, côté glacier. Dans certaines zones, il s'est créé un sillon à parois asymétriques, haut de $10 \mathrm{~m}$, côté moraine, et de $20 \mathrm{~m}$, côté glacier, séparé par quelques mètres de vide ou rempli d'eau en automne (altitude : 2680 environ).
- En été, sur certains secteurs de la rive droite, on relève des extrusions latérales de glaces qui, avec des angles de 30 à $45^{\circ}$ en plan, affouillent les moraines anciennes et rehaussent des blocs de rocher de plusieurs dizaines de tonnes. Ainsi, on a une vision extraordinaire de la formation dynamique des moraines. A notre avis, ces extrusions localisées résultent de pressions intraglaciaires hétérogènes et qui proviennent des forces en jeu : poussée du glacier et force de gravité. De cette répartition non uniforme des pressions découle des plasticités locales plus grandes, d'où les extrusions. Sur le front du glacier du Bas d'Arolla, on a même vu au printemps une protubérance longue de $12 \mathrm{~m}$ et de plusieurs mètres carrés de section. - Les stries d'érosion provenant de burinages du lit rocheux par des pierres enchâssées dans les glaces sont particulièrement visibles sur la marge proglaciaire de Findelen. L'observation des écoulements de la glace basale par les stries est semblable à des écoulements laminaires avec des lignes de courant autour des obstacles. Les stries dans la zone marginale (rive gauche) ont tendance à s'écarter du sens de l'écoulement général du glacier. Ceci pourrait être explicité par notre hypothèse émise ci-dessus.

- Le front avance avec des plans horizontaux de cisaillement remontant de 10 à $20^{\circ}$ sur la verticale et absorbe des blocs de plusieurs $\mathrm{m}^{3}$, sans créer de moraine frontale. De 1979 à 1982, il s'est épaissi fortement. De plus, il avance de façon asymétrique, c'est-à-dire sur la rive droite en été, tandis qu'en hiver c'est principalement la rive gauche qui avance. Sur la croupe rocheuse, rive gauche, une bande de glace de 2-3 $\mathrm{m}$ d'épaisseur précède de 6 à $8 \mathrm{~m}$ la masse du glacier. S'agit-il à nouveau d'un phénomène résultant de pressions localisées avec plasticité plus grande et extrusion (Fig. 5)?

Il semble qu'un plan vertical de cisaillement ne se remarque guère sur la rive gauche qui compose l'intérieur de la courbure du glacier.

\section{Incertitudes des mouvements glaciaires dans le futur}

Nul ne peut prévoir les mouvements du glacier qui résultent des précipitations annuelles et des températures estivales.

En 1982, les grandes chaleurs estivales, compensées par des précipitations importantes, ont ralenti l'avance glaciaire.

En 1983, de fortes températures estivales, combinées avec des précipitations moyennes, ont donné un sérieux coup de frein à l'avance du glacier.

L'analyse de 3 méthodes prospectives de variations possibles du climat propose un rafraîchissement pendant encore quelques années. Il s'agit :

- de l'augmentation du nombre des taches solaires;

- des oscillations climatiques relevées à Camp Century et extrapolées par série de Fourier;

- des analyses climatiques relevées sur les carottes des foraminifères du Cap Vert et extrapolées par série de Fourier.

Il est certain que depuis quelques années, on relève des avances glaciaires généralisées (50-75\% des glaciers) sur les Alpes suisses et en particulier sur les glaciers des vals 
de Zermatt et d'Hérens, qui intéressent Grande Dixence. Relevons que certains glaciers avaient atteint des positions de recul jamais observées par les vieux guides des régions concernées, par exemple Kin : altitude du front glaciaire environ $3000 \mathrm{~m}$, malgré des sommets de $4500 \mathrm{~m}$ (Mischabel). Région semi-aride! Reynaud L., géophysicien du CNRS Grenoble, se préoccupe depuis longtemps des fluctuations glaciaires en fonction des climats et des bilans de masse. Pour Findelen, il proposait en 1981, d'après le bilan de masse moyen d'Aletsch de 1946-1981, les avances suivantes :

$1982: 10$ à $28 \mathrm{~m}$ réalité : $+43 \mathrm{~m}$

$1983: 10$ à $-20 \mathrm{~m}$ réalité : $+18 \mathrm{~m}$

Dans une semblable étude interne de Grande Dixence, Rey prévoit à fin 1982 le recouvrement de la prise en fin d'été 1984, avec une hypothèse "optimiste".

A relever sur la figure 4 les formes irrégulières du front du glacier qui rendent difficiles prévisions et réalité, surtout que le mécanisme de flux glaciaires transversaux, au droit du portail, à tendance à se manifester.

\section{Recherches}

- Les glaciologues de l'EPFZ ont en 1980 mis en place 2 appareils photographiques Olympus qui ont pris 2 photos par jour, hiver comme été, du front glaciaire. Une tentative de film, par l'utilisation des photographies, vient d'être réalisée mais sans qu'il soit possible de déterminer le mécanisme d'avance du front.

- $\mathrm{M}^{\text {lle }}$ Iken de l'EPFZ a continué les mesures des pressions hydrostatiques sur des profils, ainsi que des vitesses au front et à $2 \mathrm{~km}$ en amont, mesures déjà commencées en 1973. Les résultats seront donnés dans le prochain " Journal of Glaciology ".

- Avec l'Institut de géologie de l'EPFZ, on prévoit la pose de capteurs de pressions des glaces en amont du canal et les liaisons de mesures sont prévues jusque dans les ouvrages de commande de la prise.

- En été 1982, le géographe T. Moeri (Université de Berne) a exécuté des injections de traceurs à différents endroits : 1700 à $2650 \mathrm{~m}$ du front, dans des moulins ou des trous forés jusqu'à 100 et $170 \mathrm{~m}$ de profondeur et ceci avec différents débits de fusion. Succinctement, on peut relever :

- des vitesses maximales d'écoulement des eaux de $0,5-1 \mathrm{~m} / \mathrm{s}$, lors des gros débits d'août,

- des vitesses plus faibles en juin et en septembre : section réduite,

- que ces vitesses maximales s'approchent des vitesses que l'on mesure en écoulements libres, sur des torrents à pente semblable.

- Il est regrettable que les différents phénomènes de mouvements des glaces, soit sur la rive droite, soit sur le front, n'aient pas mieux été observés, mesurés et analysés car, à notre avis, ces observations auraient permis de mieux expliquer deux grands problèmes :

- comment s'écoule un glacier?

- comment se forment les moraines?

\section{Références bibliographiques}

BEZINGE, 1972. - Sites sous-glaciaires des Alpes dans glaces tempérées. Réunion de la section française de l'International Glaciology Society: Chamonix.

BEZINGE, 1979. - Glacier d'Argentière. Captage des eaux. Analyses, remarques et propositions. Journées EDF à Finhaut (étude non publiée).

BEZINGE, PERRETEN, SCHAFER, 1969. - Phénomènes du lac glaciaire du Gorner. Symposium de Cambridge. AIHS.

BINDSCHADLER R., 1982. - Modellrechnungen zum zukünftigen Verhalten des Griesgletschers, Wallis, Revue eau, énergie, air. Cahier 4, Baden.

CHARPENTIER, COLLIOUD, VIVIAN, 1972. - Observations glaciologiques sous les glaciers d'Argentière et de la Mer de Glace. S.H.F, Section de glaciologie, Paris.

COURDOUAN, 1971. - Les ouvrages de captage et d'adduction du torrent sous-glaciaire d'Argentiaire. Cours d'eau et énergie $n^{\circ} 8$, Zurich.

ELLISTON, 1969. - Ecoulement de l'eau à travers le glacier du Gorner. Symposium de Cambridge, AlHS.

GRARD, 1971. - Essai d'un modèle explicatif des variations des glaciers par le climat. Société Hydrotechnique de France. Section glaciologique, Grenoble.

IKEN, 1980. - Vorstoss des Findelengletschers. Jahresbericht der Versuchsanstalt für Wasserbau. Hydrologie und Glaziologie der Eidg. Techn. Hochschule, 54-55.

IKEN, BINDSCHADLER, 1983. - Detailed measurements of glacier movement and subglacial water pressure, Findelengletscher, Wallis. Conclusions on sliding mechanism, characteristics of glacier bed and subglacial drainage system. In preparation (will be submitted to the $J$. of Glaciology).

LLIBOUTRY, 1983. - Modifications to the theory of intraglacial waterways for the case of subglacial ones. Journal of Glaciology; vol. 29, no. 102.

LLIBOUTRY, HANTZ, 1983. - Waterways, ice permeability a depth and water pressures at glacier d'Ar̉gentière, French Alpes. Journal of Glaciology; vol. 29, no. 102.

MOERI, 1983. - Beitrag zum Abflussgeschehen des Findelengletschers. Travail de diplôme, Institut de Géographie, Université de Berne.

OULIANNOFF, 1948. - Considérations glaciologiques relatives aux prises d'eau sous-glaciaires (Etude Grande Dixence, non publiée).

RACT, MADOUX, REYNAUD, 1951. - L'exploitation des glaciers en profondeur. La Houille Blanche, no spécial A.

REYNAUD, CORDOUAN, 1962. - Reconnaissance du thalweg sous-glaciaire de la Mer de Glace, en vue de l'établissement d'une prise d'eau. La Houille Blanche, no 2.

REYNAUD, 1959. - Prospection au glacier d'Argentière. Société Hydrotechnique de France. Section glaciologique, Paris.

REYNAUD L., 1977. - Glaciers fluctuations in the Mont-Blanc area (French Alps). Zeitschrift für Gletscherkunde und Glaciogeologie. Vol. 3, no. 1/2.

REYNAUD L., 1978. - Can the linear balance model be extended to the whole Alps. AIHS. Publ. no. 126.

ROETHLISBERGER H., 1971. - Massnahmen gegen die Ausbrüche eines Gletschersees ob Saas Balen. Schweizerische Bauzeitung, 89, Heft 40.

ROETHLISBERGER H., 1972. - Water pressure in intra- and subglacial channels. Journal of Glaciology; Vol. 11, no. 62.

VIVIAN, 1969. - La nappe phréatique du glacier d'Argentière, AIHS, Cambridge.

WAEBER, 1943. - Observations faites au glacier de Tré-la-Tête. Revue de Géographie Alpine. Grenoble.

WAEBER, 1946. - Aménagement d'une prise d'eau sous-glaciaire. Publication dans Mémoires et Documents, Grenoble.

WAEBER, 1946. Alimentation de la Grande Dixence au moyen de prises sous-glaciaires (étude non publiée). 


\section{Discussion}

$M$. le Président indique qu'après l'exposé théorique précédent a été présenté un rapport qui met en évidence le grand intérêt pour les électriciens, des études des glaciologues.

M. REYNAUD ouvre le débat; il constate l'intérêt des documents présentés par M. BEZINGE : ils permettent en effet de montrer l'état d'un glacier à une saison où les observations manquent. Le cisaillement et l'extrusion observés sont caractéristiques de glacier en avance rapide, on retrouve des formes analogues sur la rive gauche du glacier d'Argentière; mais l'extrusion n'est peut-être pas la seule explication de lavancée de la glace, toujours extrêmement noire et chargée dans ces zones. C'est peut-être simplement lablation différentielle qui joue, laissant en place les portions de glace très recouvertes. Des mesures sont certainement nécessaires.

M. BEZINGE maintient son explication, il cite cependant l'exemple d'un petit glacier, présentant un cisaillement long d'un $\mathrm{km}$, qui pourrait se prêter parfaitement à une telle étude. Il en profite pour rappeler les efforts effectués sur le glacier de Findelen, par l'Université de Berne (mesures de temps d'écoulement) et l'équipe de Zurich (mesures d'avancement par photographie et mesures de pression de poussée de la glace) avec mesures hydrostatiques.
Le conférencier confirme à M. Le Président que la prise qui actuellement ne fonctionne pas en sous-glaciaire, ne sera plus visitable, lorsqu'elle sera recouverte par les glaces.

A ce propos, M. ROBERT demande dans quelles limites la prise est protégée de l'avancée des glaces.

M. BEZINGE juge la protection suffisante jusqu'à une épaisseur de glace de $60-80 \mathrm{~m}$. Il pense, que de toute façon, pour des épaisseurs supérieures, les forces de pression rendront étanches la couche basale et compromettraient le captage.

M. BERNARD demande si des mesures (sismiques par exemple) ont été faites afin de reconnaître le profil du glacier à l'amont de la prise. Des sondages ont été effectués il y a 20 ans au niveau du collecteur à $2 \mathrm{~km}$ en amont. Ils ont montré une certaine dissymétrie entre rive droite (moraine de fond) et la rive gauche où le lit rocheux est plus bas (épaisseur de glace plus grande!). Ce phénomène a été recoupé par $\mathrm{M}^{\text {lie }}$ IKEN de l'E.P.F.Z. lors de ses derniers sondages.

M. Le Président remercie M. Bezinge. 